\title{
Téoros
}

Revue de recherche en tourisme

\section{Culture professionnelle et métiers du tourisme sportif de montagne}

\section{Jean Corneloup et Philippe Bourdeau}

Volume 20, numéro 3, automne 2001

URI : https://id.erudit.org/iderudit/1071630ar

DOI : https://doi.org/10.7202/1071630ar

Aller au sommaire du numéro

Éditeur(s)

Université du Québec à Montréal

ISSN

0712-8657 (imprimé)

1923-2705 (numérique)

Découvrir la revue

Citer cet article

Corneloup, J. \& Bourdeau, P. (2001). Culture professionnelle et métiers du tourisme sportif de montagne. Téoros, 20(3), 32-44.

https://doi.org/10.7202/1071630ar d'utilisation que vous pouvez consulter en ligne.

https://apropos.erudit.org/fr/usagers/politique-dutilisation/ 


\section{Culture professionnelle et métiers du tourisme sportif de montagne}

\section{Jean Corneloup ef Philippe Bourdeau}

The authors of this article have taken an historical perspective to analyse the dynamics involved in a professional approach to nature sports. These dynamics show a disparity between the practice of antateur and professional mountain sports. Professional training tends to open up new levels of competence, very different from those of the amateur: Aggressive marketing has forced the development of various products that stresses professional achievements. Trainers, field guides, kayak instructors for example have often played an important part in forcing innovations, but they only represent a small portion of the people presently involved in tourism and mountain sports. The market has shifted to other groups and other forms of development that changes the rules of the game. Nowadays it is more a question of becoming a competent sportsman rather than an outstanding one.
$\mathbf{N}$ ombreux sont les écrits qui portent sur l'analyse des métiers sportifs. Bien souvent, les approches s"inscrivent dans une perspective économique : le propos consiste à présenter le chiffre d'affaires du secteur touristique, les caractéristiques des métiers, les évolutions à venir et les profils professionnels attachés à ces fonctions. Le présent texte cherche à développer un point de vue différent dans l'étude des pratiques professionnelles. Considerant que les métiers sportifs de la montagne sont des pratiques culturelles qui expriment une certaine manière de s'engager dans les usages et la gestion des espaces de nature, nous souhaitons apporter quelques éléments de connaissance et de réflexion pour mieux appréhender les enjeux qui traversent la dynamique de ce champ (au sens de Pierre Bourdieu, 1987) ou de ce marché au début du XXI $\mathrm{XI}^{e}$ siècle.

Pour traiter cette thếmatique, l'étude des principes culturels des métiers concernés permettra de repérer les éléments qui structurent les logiques d'action professionnelle. La prise en compte de ces principes servira de référent pour aborder l'historique des métiers sportifs de la montagne et pour en décoder les changements significatifs. Mais la connaissance des métiers ne peut se contenter d'une approche élémentaire. L'étude globale de ces métiers impose une lecture très complète qui intègre la dimension territoriale. Les dimensions culturelles et politiques des pratiques professionnelles sont davantage compréhensibles quand les métiers sont étudiés par rapport à leur manière de participer à des formes touristiques identifiables.

\section{Les pôles culturels des métiers sportifs de la montagne}

Quatre pôles structurels participent à la déclinaison des usages professionnels de ces acteurs touristiques. Ils permettent d'introduire les différentes dimensions de l'action professionnelle telle qu'elle se construit dans le quotidien de la pratique. Souvent négligée, cette approche se présente pourtant comme un cadre d analyse fécond pour rendre compte de la réalité vếcue dans l'action quotidienne. Les pôles $s^{*}$ emboîtent ainsi mutuellement pour révéler les dimensions culturelles des métiers concernés en fonction des marquages pédagogiques, sportifs, organisationnels et sociaux identifiés. Concrètement, les cultures professionnelles en situation révèlent la présence de ces quatre pôles: logique pratique, sportive, organisationnelle et sociale.

\section{La logique pratique}

On s'interesse ici à la manière dont le professionnel développe une relation d'échange avee un ou des clients dans le cadre de la pratique : quelle est la forme pédagogique envisagée ? Comment s'effectue la transmission de l'information et des contenus d'action? Quels types de relations se développent entre les différentes parties ? Peut-on parler d'éthique de l'action? On ne peut négliger la prise en compte de ce premier niveau de l'action professionnelle dans le cadre des loisirs sportifs de nature. Un contrat d'échange se construit entre le professionnel et le client. Il permet l'établissement d'une communication autour de laquelle se définissent les termes de l'échange, les biens echangés, le coût de lâ prestation, 
les engagements pris par les uns et les autres ainsi que les formes de régulation envisagés. Au-dela de la prestation définie par le contrat professionnel, c'est dans l'interaction sportive que se construisent les liens. En fonction des expériences vécues dans l'action, des attentes et des satisfactions observees, une culture professionnelle sera evalué et elle entratnera peut-être une fidélisation de la clientèle. La question de la durabilité se perçoit déjât sur le plan des formes d'encadrement développées en fonction des profits attendus par les différentes parties présentes. Dès lors, les études réalisées sur les services sportifs (Pigeassou, 1997 ; Desborde, 1999) ainsi que celles en anthropologie cognitive (Ria, 2001 ; Gal et Durand, 2002) $\mathrm{s}^{*}$ inscrivent totalement dans ce pôle pour mieux comprendre les logiques d'action professionnelle.

\section{La logique sportive}

Le rapport aux pratiques ne va pas de soi. En fonction des preferences culturelles, le choix des sports et des modalités de pratique activées dans un cadre de service ne sera pas identique. Les territoires professionnels utilisés et les territorialités d'action produisent un marquage culturel. Des significations géographiques différentes emergent quant â la manière de faire usage et de marquer l'espace d'action (Bourdeau, 199 lb). Selon les technologies utilisées et les aménagements réalisés, le rapport ầ la nature (Vachée, 2000) et à l'objet sport divergent. Les identités professionnelles se révèlent dans la lecture du traitement * didactique ses pratiques effectuées. Un style culturel émerge ; il précise la conception que le professionnel a de la pratique et l'engage dans une lecture sportive bien particulière. En fonction des propriétés physiqueset symboliques des pratiques retenues par les acteurs, le rapport à l'objet sportif, à l'espace d'action et au client est différent. Une vision du monde et un rapport particulier à la culture sportive légitime se développent. Ces marquages ont une certaine importance dans la compréhension des cultures professionnelles. Le traitement et le choix des pratiques situent socialement et professionnellement le prestataire. En s'inspirant des écrits de
Christian Pociello (1995) et de Pierte Bourdieu (1987), on identifiera un espace des positions professionnelles en fonction des relations engagées avec les pratiques sportives. Des stratégies sont présentes, dépendantes du positionnement des acteurs dans l'espace social et professionnel des pratiques sportives. Enfin, en fonction de l'ethique professionnelle retenue, des différences apparaissent dans le contrat sportif annoncé et les finalités culturelles poursuivies.

\section{La logique organisationnelle}

Chaque métier s'inscrit dans une organisation qui prend différentes orientations en fonction du style retenu, Selon l'implantation territoriale, les stratégies marketing choisies, les types de management développés et d'éthique défendue, des différences emergent entre les acteurs du monde professionnel. Pratiquer un métier engage l'individu dans l'économie territoriale et participe à la déclinaison d"une identité professionnelle comme facteur d'engagement local et social. Selon les formes de décision prises (autoritaires ou participatives), la conception du métier (rentabilité, passion, logique public ou privé), la relation au client (commerciale, financière, temporelle) ou encore la participation ou non de l"entreprise au dynamisme local, la forme de développement de la structure ne sera pas équivalente.

\section{La logique sociale}

Considérant que le style professionnel n'est pass séparé des modes et des styles de vie de l'acteur, la prise en compte d'un quatrième niveau permettant de décoder les relations qui existent entre le métier et la vie sociale de l'individu semble nécessaire. En effet, la vie familiale vécue, les stratégies concernant le style de vie, les valeurs affichées, la formation suivie ainsi que les pratiques culturelles préférées participent à la définition de l'identité culturelle et professionnelle. La manière dont l'individu s'engage dans la vie locale et construit ses relations de proximité ne peut être sous-estimée. Le rapport au lieu, à la nature et à la vie sociale participe à la compréhension de l'identité sociale autour de laquelle se construit son engagement professionnel. Saisir cette logique sociale nécessite de s"intéresser aux formes d'echanges qui prennent corps dans le quotidien de la vie à partir de l'identification des expériences sociales vécues. Selon que le professionnel est perçu comme un * local $*$ ou un \& étranger $\%$, son ancrage local se fera d'une façon différente.

Le détour par cette approche structurelle permet d'identifier des variables actives qui participent à la saisie des cultures professionnelles attachées aux métiers sportifs. Une grille de lecture peut être elaborée (figure 1) pour faire une comparaison entre métiers et saisir des différences pertinentes entre cultures professionnelles. La connaissance de ces pratiques nécessite de s'intéresser aux situations vécues par les professionnels pour comprendre ces logiques d'action. Tout un champ d'observation serait à développer pour saisir ces interactions professionnelles qui participent à la déclinaison des formes de métier. Les approches ethnométhodologiques tournées vers le décryptage des singuląrités de l'action (Quéré, 2000) et des expériences professionnelles seraient tout à fait justifiées (Bernoux, 1996).

\section{D'une génération à I'autre}

Notre propos consiste à montrer, à partir d'une perspective historique, la présence d'une fragmentation qui contribue à produire des changements dans la dynamique des métiers sportifs de la montagne. Des enjeux considérables émergent, portant sur les visions de la montagne, du sport, du métier et des clients. Nous faisons l'hypothèse qu'à la suite de la première génération de métiers qui a structuré ce champ professionnel pendant un siècle, une deuxième génération en émergence repositionne considérablement la dynamique de ce secteur. Au cours de la période 1980-2000, une nouvelle culture est apparue modifiant considérablement les usages historiquement définis. Brièvement, on peut présenter les différences qui opposent les deux styles de métiers dans la 


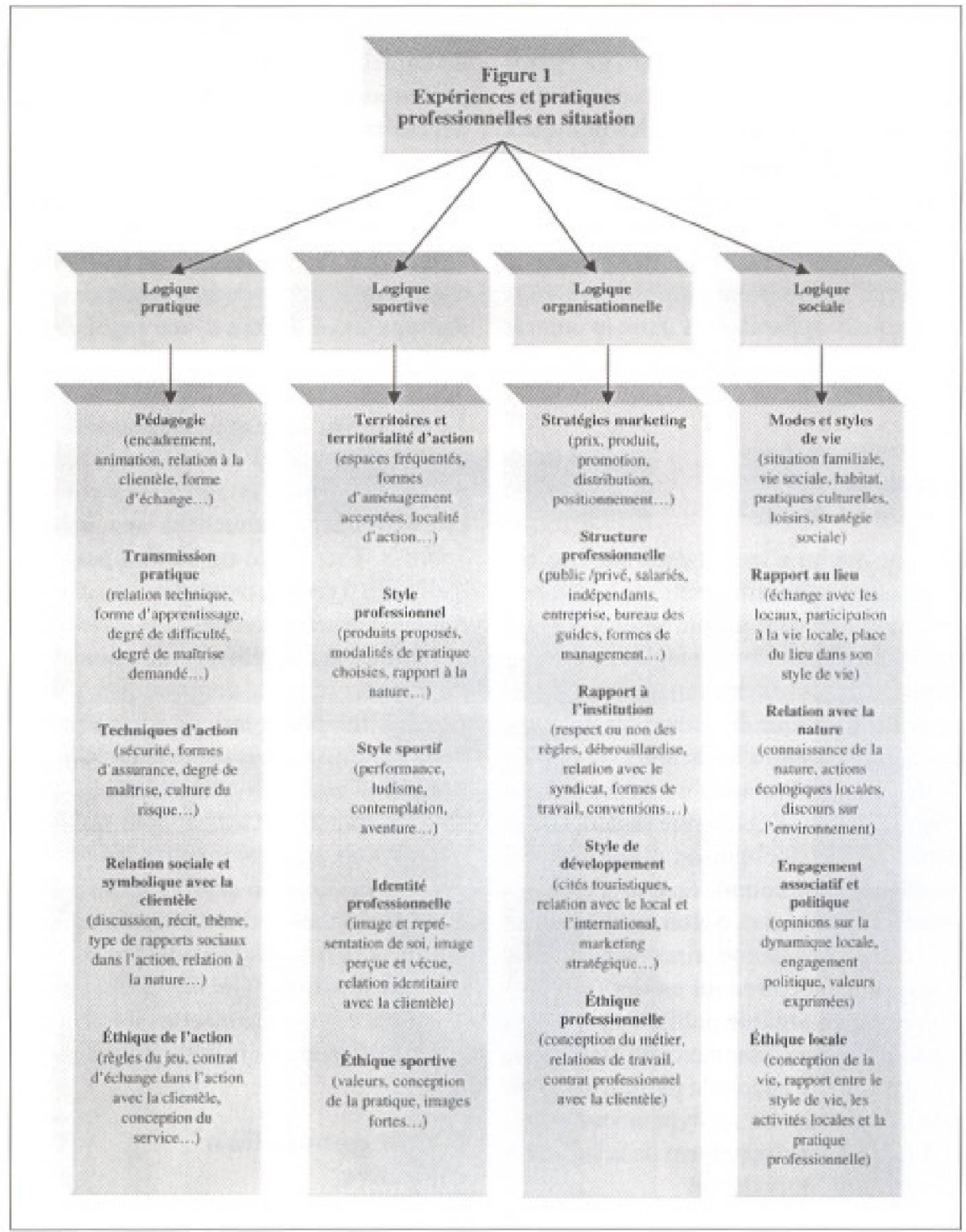

continuité des changements qu'Alain Loret (1993) avait identifiés globalement dans le domaine des métiers du sport en France.

\section{La culture professionnelle moderne (première génération)}

Cette culture s'est affirmée au cours du $X^{c}$ siècle (1930-1960) avec la a sportivisation $*$ de l'alpinisme et l'ouverture du métier de guide de haute montagne (De Bellefon, 1997). Dans le même temps ou avee un décalage de quelques décennies, d'autres métiers viendront s"inscrire dans ce modèle à l'image de la plupart des pratiques sportives de nature encadres : moniteur de ski, moniteur de un processus d'ancrage a une pratique sportive de référence (alpinisme, ski, kayak...) qui commence bien souvent dans l'enfance (Corneloup, 1999a). Dans un deuxième temps, une formation professionnelle est réalisée, sous couvert de Jeunesse et Sport, au sein de l'ENSA' ou des CREPS ${ }^{2}$. Une fois l'entrée effectuée sur le marché des métiers de la montagne. on observe une adaptation faible a la dynamique du marché. Une logique de la reproduction est observable d'une génération a l'autre. Les variations dans les formes d'encadrement et d'organisation sont minimes au cours du temps. L'eneadrement proposé repose sur un aménagement léger en direction d'un public de specialistes, sportifs pour la plupart. En allant plus loin, le détail de cette culture peut être présenté selon le modèle de la figure I:

- Sur le registre des formes d'encadrement, l'uspect engagé de la pratique, en réference a la culture a alpin-cafiste $w$ dominante (Corneloup, 1999a), justifie la mise en place d'une pédagogie directive autour d"un encadrement strict. Dans un souci de réussite et d'efficacité, des groupes de niveaux sont définis permettant de différencier hierarchiquement les pratiquants. Une transmission technique domine sur un plan tant securitaire que sportif, visant à diffuser les codes culturels et les savoirs initiatiques de la pratique. En alpinisme, par exemple, Oivier Hoibian (2000) evoque la domi nation d'une conception techniciste pendant de nombreuses décennies, Dans cette perspective, l'encadrant se situe au centre de l'action pédagogique. C'est un modèle, une référence et une fïgure emblématique ; ill est admiré et respecté (Bourdeau, 1992). II domine la relation d'echange sur le terrain sportif. On est en présence d'une pratique professionnelle qui s'inserit dans la durée, qui valorise le progrès et qui justifie la présence d'apprentissages longs et fastidieux en vue d'obtenir une attestation de niveau (en ski) ou de réussir une belle course en montagne (en alpinisme). L'échange symbolique est construit autour de récits sportifs et héroïques ainsi que sur la symbolique de l'épreuve 
(Jeu, 1984). La présence d'un temps long passé sur le terrain dans l'intimité d'une relation de proximité, à cause de la durée de la pratique, est une occasion de développer des relations humaines fortes. Enfin, il faut noter la présence d'une éthique de la responsabilité et de la transmission, ctant donné que l'encadrant est directement engagé dans la gestion de la sécurité et qu'il participe grandement a la definition des règles du jeu dans l'action contractée entre les différentes parties en présence. Les matériaux historiques mis en perspective par Olivier Hoibian $^{3}$ (2000) illustrent bien les fondements de cette approche.

- Sur le plan de la dominante sportive. cette première génération de métiers $s{ }^{*}$ inscrit prioritairement dans les teritoires de la haute montagne, sur les sites majeurs de pratique et dans les stations de sports d'hiver de la * première période $\rightsquigarrow$. C'est la montagne majestueuse, dominante et turbulente qui est privilégiée, d où la priorité donnée à un aménagement léger et discret. Dès lors, il $s$ agit de limiter au maximum les formes d'aménagement, que ce soit pour le balisage des chemins, la conception des topo-guides, des points d'ancrage apposés dans les voies de montagne ou du style des refuges alpins. Une même logique apparait : elle valorise l'engagement de la personne et la nécessité de prendre des professionnels pour s'aventurer sur ce territoite. La creation d'ecoles d'escal ade comme propédeutique à la formation et à l'initiation s'inscrit dans cette logique très sportive. En effet, le traitement professionnel des pratiques est très faible. L'optique est de se rapprocher et de reproduire le plus possible le modèle de la culture sportive défini socialement. Dès lors, l'encadrement est marqué par la présence d'une logistique lourde et contraignante. De fortes contraintes temporelles, logistiques, météorologiques sont présentes. Bref, la culture sportive énergétique, ascétique, ascensionnelle, rigoureuse et dramatique est fortement ancrée dans les mentalités professionnelles autour d'une éthique républicaine et civique ${ }^{4}$. Par la pratique se transmettent les valeurs de l'effort, de la rigueur, de la maitrise de soli, de la soumission à l'expert et à l'ordre, tout en valorisant la prise d'initiatives réelles ou simulées. On confirme ici les propos que Bernard Debarbieux (1995) développe dans son analyse du tourisme de montagne moderne construit sur le modèle de la culture sportive.

- En ce qui concerne les formes d'organisation, le modèle dominant est celui de la petite structure de plein air qui fonctionne de manière très structurée et pyramidale. A l'image des organisations historiquement constituées (le bureau des guides et l'ecole du ski français), il faut retenir la forte présence d'une gestion administrative développée à la fois par les institutions nationales (Club Alpin Français, Federation Française de la Montagne, UCPA ${ }^{5} .$. ) et par les acteurs locaux. En effe $\iota$ les a anciens $x$ et la tradition participent largement à la definition et à la diffusion de cette culture managériale patrimoniale (Loux, 1988) au sein de laquelle des stratégies de contrôle structurel sont mises en place par les acteurs (Bourdeau, 199 lb). Dès lors, il faut insister sur la présence dominante des instances politiques, étatiques et associatives dans le management de ces structures à l' image de Jeunesse et Sports (ENSA), des syndicats professionnels, des federations et des associations sportives $\left(\mathrm{CAF}^{n}\right.$, UNCM $\left.{ }^{7} . ..\right)$. Dans cette perspective, le bassin de clientèle est fourni par la culture et les clubs sportifs des pratiques de la montagne. Les actions et les stratégies marketing ne sont donc pas nécessaires tant le système donne l'impression de fonctionner a naturellement w... La dominante va alors à une culture professioninelle marqués par une empreinte publique et associative qui valorise un modèle de développement rếpublicain, educatif et patriarcal (Corneloup, 2000). Une logique de la reproduction domine, où il sagit de conserver les acquis historiquement obtenus. Mais, en même temps, il faut souligner que ces professionnels sont des independants au sein de structures professionnelles qui fonctionnent historiquement sous une forme associative. Cette situation leur permettra, au cours du temps, d'augmenter le jeu avec la règle étatique, le guide ou le moniteur étant bien souvent un débrouillard $*$ qui se joue des normes et des règles étatiques...

- Enfin, la compréhension de la culture professionnelle issue de cette première génération serait incomplète sans la prise en compte de la logique sociale autour de laquelle se construit l'ancrage professionnel. Si, jusque dans les années 1940, les professionnels de la montagne sont presque exclusivement issus du monde paysan (De Bellefon, 2000; Loux, 1988), l'êmergence de professionnels d'origine urbaine à la recherche d'une intégration locale au monde et à la culture montagnardes s affirmera ensuite comme une tendance lourde. Un ancrage territorial est recherché autour d'une identification aux valeurs a montagnardes $x$ (le chalet $\alpha$ anto-construit $\%$, la chasse, la coupe de bois...). Cette aspiration ane anontagnite $*$ acquise couvre une dimension de quête de liberté et d'expression de soi en dehors des contraintes urbaines et sociales. Bien souvent, le professionnel cultive une vision décalée et valorise un style de vie de type $\alpha$ plein-air $*$. La nature est considérée comme un refuge et une école de vie ; elle se mérite et le professionnel entretient cette vision moderne de la nature. Enfin, comme cela a été souligné précédemment, la formation est très classique et repose sur l'assimilation d'une culture technique, sécuritaire et institutionnelle qui vise à effectuer un transfert entre le savoir, le savoir-faire et le savoir-être acquis lors de la pratique sportive et ceux qui sont nécessaires à l'activité professionnelle (Bourdeau, 1991 b). Bref, un passage de l'éthique sportive à un ethos professionnel s'effectue via l'institutionnalisation d'un diplôme d'État hérité de la double loi fondatrice de fevrier 1948 sur les guides de montagne et les moniteurs de ski. Ajoutons à ce constat la présence d'un certain decalage entre, d'une part, la forte valeur ajoutée symbolique liée à l'identité professionnelle et, d'autre part, la valeur ajoutée économique à la fois assez faible et incertaine de la pratique professionnelle. 
La culture professionnelle post-moderne (deuxième génération)

Une culture professionnelle post-moderne a émergé au cours des années 1980, bouleversant le champ des métiers sportifs de la montagne. En quelques années, une autre logique d'action est née, déstabilisant l'ordre professionnel qui structurait jusqu'à cette période le marché des sports de pleine nature. Ce phénomène participe à l'affirmation de nouveaux modèles d'usages touristico-sportifs de la montagne - à la fois plus ludiques et plus diversifiés et de nouveaux modèles de développement territoriaux plus diffus et plus ouverts avec, notamment, une multiplication des pratiques et des terrains de jeu concernés (Bourdeau, 1998).

En premier lieu, ce sont les conditions d'access aux métiers sportifs de la montagne qui se transforment. Alors que depuis une quarantaine d'années une culture sportive dominante assez stable marquait les professionnels de son empreinte, l'émergence de nouveaux référents culturels s'accompagne d'une modification des codes d'entrée dans la sphère professionnelle. S'imprégnant du mouvement californien, * fun », ludique et hédoniste (Midol, 1992 ; Loret, 1995 ; Corneloup, 1999a) qui se propage dans le monde des sports de nature, une nouvelle génération de pratiquants s'engouffre dans cet univers et s'approprie d'autres codes culturels que certains utiliseront comme capital à usage professionnel. Pour d'autres, l'entrée dans la culture professionnelle tend a se couper de l'univers des pratiques sportives dans la mesure où s'intègrent directement les dispositifs émergents de pré-formation et de formation. De plus, l'université avec ses formations diversifiées (UFR STAPS ${ }^{\circledR}$, filières management, écoles de commerce, IUP $^{9}$ et DESS ${ }^{10}$ tourisme, loisirs et sports...) et le milieu entrepreneurial se présentent comme des forces structurelles qui s'efforcent de prendre le relais des acteurs de la première génération (fédérations, Jeunesse et Sports, ENSA, UCPA ... ). L'innovation dans la formation se situe au niveau de cette reconfiguration du marché pour mieux réppondre à la dynamique culturelle du tourisme de montagne. La tendance à la privatisation des formations ne manque pas non plus de modifier les cultures professionnelles. Si, pendant une dizaine d'années (1980-1990). les acteurs dominants de cette a nouvelle Economie $*$ des sports de montagne étaient encore issus de la première génération de professionnels et abricolaient * leur immersion dans le monde commercial, les années 1990-2010 annoncent l'affirmation conquérante d'une deuxième génération de métiers. Au sein de cette deuxième génération, les activités d'encadrement sportif $\mathrm{n}$ 'occupent plus une place préppondérante face à la montée en puissance des métiers de l'animation, du développement, de l'innovation et du management des sports et des produits de montagne.

L'identité et le statut de ces professionnels se transforment quand les prestataires et les entrepreneurs du loisir sportif de nature marquent de leur présence le marché, en développant des produits pour des segments de clientèle choisis en fonction de stratégies marketing définies. Le monde du plein air quitte son univers anti-commercial qui le caractérisait jusque-là (Majastre et Decamp, 1988) pour s'engouffrer dans celui du marketing et de la réussite entrepreneuriale. La concurrence entre les différents opérateurs monte en puissance, relayée par la montée des enjeux territoriaux quand les villes, les départements et les régions se disputent le marché des destinations touristiques. Si autrefois la logique de la reproduction dominait, aujourd'hui le rythme de gestion s'accelère et valorise l'innovation et la culture * tendance $\%$. Les acteurs ont l'obligation de s'adapter à la dynamique du marché qui est sans cesse traversée par des mouvements de différentes natures (économique, commerciale, technologique, culturelle, médiatique...). En quelques traits, les contours de cette deuxième génération de métiers peuvent être esquissés à partir des quatre polarités culturelles déjà proposées (figure 1):

- En ce qui concerne les formes d'encadrement, les logiques pédagogiques évoluent et tentent de répondre aux attentes montantes de la culture postmoderne" ${ }^{\| 1}$. L'encadrement s'assouplit, les contraintes diminuent et les relations prestataires-clients se personnalisent. donnant la priorité à l'animation. La rigidité n'est plus de circonstance ; il faut séduire, attirer et faire plaisir. La prestation doit valoriser la culture et la transmission de sensations au détriment de la technique, tout en favorisant le plus possible l'expression d'émotions primaires. L'apprentissage est réduit en durée et doit faciliter la rapidité de la mise en action. Les tendances lourdes relevées par Christian Pociello (1985) semblent s'appliquer parfaitement au marché des sports de montagne dans cette priorité accordée aux faibles contraintes, aux petits groupes et à l'interactivite dans la rencontre construite entre le pratiquant et les espaces d'action. Le cadre professionnel, issu de la culture technique, s'efface pour laisser au client la possibilité d'être l'acteur de son action. La technique sécuritaire (mise en place autrefois par le guide, par exemple) et dominante, jusqu'à présent, cède la place à la technologie sécuritaire à l'image des * via ferrata $*$ et des parcs aventure ${ }^{12}$. La prise de risque simulée produit une valeur ajoutée émotionnelle pour le plus grand plaisir des consommateurs. Une mise en récit ludique et émotionnelle prend forme dans la propagation des histoires, des slogans et des propagandes commerciales. La mise en scène devient de plus en plus néccessaire pour renforcer l'esthétique de l'échange (Maffesoli, 1993). La personnalisation des apparences s'impose, accréditant la place légitime appropriée par la culture des services. Enfin, contrairement à la culture moderne précédente, la règle d'action définie par l'encadrant s'efface pour laisser la place à une co-production et à un co-pilotage. Le client devient plus participatif et actif dans la déclinaison de son programme d'action, ce qui a pour effet de modifier les cultures professionnelles et les relations prestataires-clients. Une culture de l'émotion et de la sensation prend ainsi le relais de la culture sportive d'antan à l'image de ce que l'on observe dans la société globale (Foreseen, 1999a).

- Dans le domaine des pratiques sportives proposées, les logiques profession- 
nelles se transforment quand la culture sportive s'efface au profit d'une culture ludique marquée par un traitement entrepreneurial des pratiques. Les espaces de pratique valorisés se modifient lorsque les ex-territoires interstitiels (fonds de valles, petits rochers, espaces de l'ombre... ) et les vallées deviennent les lieux d'excellence moyennant une recomposition des sites, des aménagements, des trajectoires et des lieux d'action. A l'échelle de la plupart des massifs de montagnes, ce phénomène se traduit par un singulier rééquilibrage des polarites touristiques du fait de la revalorisation des zones de vallée par rapport aux zones d'altitude. Alors que, dans la vie touristique montagnarde traditionnelle, la dichotomie vallée / altitude marque aussi une limite entre une passivité dévalorisée (en bas) et une sportivité valorisée (en haut), les « nouveaux a loisirs sportifs se pratiquent de manière privilégiée dans les fonds de vallées ou à proximité immédiate, tout en en se rapprochant de l'espace organisé des villages et des stations (Bourdeau, 1998).

Ajoutons que ce mouvement est amplifié par la présence d'une urbanisation et d'aménagements lourds des sites qui va de pair avec la * technologisation $*$ forte des pratiques sportives de nature. Les stations de sports d'hiver ne sont pas en reste, qui, sous l'effet de nombreux facteurs, sont entrées dans une nouvelle période de gestion de leur territoire et des pratiques ${ }^{13}$. Une autre difference se perçoit au niveau de la gestion de la clientèle. Si autrefois la relation s'inserivait dans la durée, aujourd'bui la gestion des flux s'impose pour accroître la valeur ajoutée des produits sportifs de nature. A l'image des stations de sports d'hiver, la rentabilité est liée à la fréquentation et au débit, euxmêmes dépendants de la fidélité de la clientèle qui se mesure aussi en termes de débits aux remontées mécaniques. La valeur du produit n'est plus dépendante de la culture sportive classique, mais des capacités des professionnels à proposer des produits de qualité en phase avec la diversité des cultures sportives de la montagne. Dès lors, la valeur ajoutée apportée par les prestataires et les entrepreneurs devient centrale, nécessitant une segmentation de plus en plus poussée des clientèles et une diversification de la gamme, La richesse de ce nouveau marché est attachée aux compétences marketing de cette deuxième génération de professionnels pour apporter de la valeur ajoutée dans la conception $\mathrm{d}^{+}$événementiels, de défis, de scénarios thématiques pour les entreprises et les autres clientèles potentielles. L'innovation s'impose partout, que ce soit sur le plan des technologies d"action, des sites artificiels de pratique (parcs aventure, a accrobranche $n_{4}$, snowpark $n_{2 . .}$ ), des circuits à thème et des éléments technologiques qui agrémentent sans cesse les nouvelles formes de motricité ludique. La culture du produit irradie l'univers professionnel lorsque la réussite professionnelle repose sur la capacite a traiter les pratiques et les objets sportifs en fonction de stratégies de marketing et de développement choisies. Bref, la logique de la reproduction propre à la culture sportive de la première génération est bien éloignée de cet univers en gestation. Une éthique marketing et entrepreneuriale s'impose et renouvelle les formes de gestion des pratiques sportives.

- Les formes d'organisation ne sont pas en reste lorsqu'elles prennent leurs distances avec une gestion publique, associative, bénévole et amateuriste. Une gestion entrepreneuriale semble s'imposer, modifiant les cultures managériales de la première génération. L'arrivée de tours opérateurs, de grands groupes touristiques à l' image du Club Méditerranée, de structures privées et de financeurs (Compagnie des Alpes...) al a recherche de profits capitalisables entraîne une professionnalisation accrue des métiers de la montagne. Le développement considérable des Sociétés d'Économie Mixte au sein des stations touristiques à la fin du XX siècle révèle un tel changement dans le mode de gestion (Vlès, 1996). Aujourd'hui, les compétences ne sont plus issues d'une culture sportive acquise sur le terrain, mais sont directement liées à des connaissances issues des sciences de la gestion, du marketing, du droit, de la comptabilité analytique... Une gestion globale et fi- nancière du produit s"impose, justifiant le rôle central et moteur occupé par le concepteur de produit et le chef de projet au détriment du technicien et du cadre sportif. La segmentation des fonctions est populaire au sein d'equipes de projets touristiques. Les principes du marketing stratégique sont appliqués à la gestion des produits sportifs en fonction de l'orientation-marché définie (Lambin, 1998). La position marketing sur le marché des produits sportifs organise les stratégies d'action quand les questions de fidélisation, de cycle de vie des produits, d'innovation et de cultures tendances $\times s$ 'imposent pour répondre aux évolutions du marché et aux attentes fines de la clientèle. Dans ce secteur en pleine expansion, la petite entreprise de plein air genre a high tech $*$, orientée vers l'innovation de produits, le conseil en développement, la création de nouveaux services... a des cartes à jouer face aux grandes structures touristiques et entrepreneuriales. Par la maîtrise des technologies de l'ingénierie touristique et de la communication post-moderne (Internet, marketing de l'innovation, culture \& icranique ${ }^{1+}$,...) réalisée par les professionnels de la deuxième génération, tout un ensemble de services innovants verront le jour dans les années à venir, modifiant là culture professionnelle des métiers de la montagne.

- Enfin, on peut observer que les logiques sociales des professionnels se transforment quand ceux-ci pensent différemment leur identité au travail en fonction de leur style de vie. Ainsi, contrairement à la premièré génération observée, la culture montante est la culture urbaine, issue directement du mouvement « fun $»$, de la culture entrepreneuriale et des formations universitaires «professsionalisantes ». Les référents culturels, historiquement construits autour de la nature-refuge, du sport * alpin-cafiste ${ }^{15} \infty$ (Corneloup, 2000) et de la communauté montagnarde s'effritent pour laisser la place à la culture des managers à la recherche de reconnaissance entrepreneuriale. Un nomadisme professionnel est perceptible quand la destination professionnelle choisie est modulable 
en fonction des opportunités et des chances de réussite. Cette territorialité d'action professionnelle s'applique aussi aux acteurs de la première génération des métiers de la montagne. Le massif du MontBlanc et la vallée de Chamonix font ainsi l'objet d'une polarisation croissante de l'activité professionnelle des guides de haute montagne français et européens (Celard, 1999). Par ailleurs, la stratégic de l'ombre, de la débrouillardise et de la transgression n'est plus valorisée ; ces nouveaux entrepreneurs épousent les valeurs de la normalité et de la culture légitime et libérale quand l'espace touristique et la nature se concoivent comme des ressources externes en vue de profits attendus. Depuis la fin des années 1980 , plusieurs agences de voyages d'aventure ont ainsi etté fondées ou co-fondées selon les règles de l'art (licence d'agent de voyage...) par des guides de haute montagne.

La nature devient un produit et le style de vie dominant de ces professionnels de la deuxième génération s'urbanise et se normalise. Pour répondre aux exigences montantes du marché, la formation repose sur la culture de la compétence, du projet marketing et de l'ingénierie du produit, dispensée dans les formations entrepreneuriales. Un autre univers se profile, modifiant considérablement les logiques professionnelles d'antan, y compris au sein des métiers les plus anciens. A titre d'exemple, l'étude de Serge Celard (1999) sur les pratiques professionnelles des guides de haute montagne montre très bien une population de guides en exercice de plus en plus d'origine citadine $(53 \%$ sont nés hors de départements de montagne), diplômés (40 \% ont un niveau d'étude supérieur au bac contre $11 \%$ en 1985), * indépendants ( $55 \%$ ne sont pas affiliés à une compagnie contre $40 \%$ en 1985) et monoactifs's ${ }^{16} *(23 \%$ de guides * monoactifs contre $16 \%$ en 1985).

\section{Espaces de pratiques, métiers sportifs et recompositions touristiques territo- riales: l'exemple de la vallée de Vallouise}

Le cas de la Vallouise, principale vallée touristique du massif Oisans-Ecrins, il- lustre très bien le phénomène de * repolarisation w des territoires touristiques qui se joue entre, d'une part, les sites, les itinéraires, les espaces, les stations de pratique et, d'autre part, la structuration locale des métiers sportifs de la montagne. Alors que jusqu'au milieu des années 1980 le seul bureau des guides de la vallée était celui de la Compagnie des guides de l'Oisans, installe à Ailefroide à 1500 mètres d'altitude, de nouvelles structures professionnelles vont peu à peu s'implanter de plus en plus en aval : tout d'abord à Vallouise (alt. $1.100 \mathrm{~m}$.) et aux Vigneaux (alt. $1000 \mathrm{~m}$.) au début des années 1990, puis à l'Argentière-laBessée au milieu des années 1990 (alt. $970 \mathrm{~m}$.). Ces structures (Diabolo Gyr, Azimut, Odyssée...), si elles portent toujours le nom de a bureaux des guides $s$, présentent une triple caractéristique qui les différencie nettement de leur aînée d'Ailefroide :

- Ce sont des structures à caractère commercial et non plus à caractère syndical (au sens où une compagnie de guides organisce en bureaux est d'abord un syndicat professionnel de vallée ou de massif, lui-même composante du Syndicat National des Guides).

- Elles sont orientées vers une pluriactivité sportive fondatrice d'une mixité professionnelle qui va bien audelà de la cohabitation - classique depuis le début des années $1980-$ entre guides et accompagnateurs en moyenne montagne: cela, dans la mesure où elles regroupent aussi des brevetés d'État d'escalade, de parapente, d'eau vive, de spéléologie, voire des * mushers $x . .$.

- Elles sont mobiles dans l'espace et le temps : déménagements intra ou inter-communaux, réorganisations, restructurations et changements de dénomination sont très fréquents ; de plus, leur ancrage territorial est très relatif, comme le montre le transfert aux Houches (vallée de Chamonix) du bureau des guides « Odyssée $*$ après quelques années de fonctionnement à l'Argentière-la-Bessé.
Le rythme de création de ces structures professionnelles à caractère commercial s'articule étroitement avec le développement de nouvelles pratiques et de nouveaux espaces de pratique (aire de parapente, site d'escalade, itinéraire de canyoning, parcours de rafting dans la seconde moitié des années 1980 ; « via ferrata $\$$ et caseade de glace dans les années 1990). Pour des raisons d'accessibilité, voire de * visibilité $n$, (site d'escalade, de parapente, « via ferrata $\% . .$. ), ou encore pour des raisons hydro-topographiques (parcours de sports d'eau vive, de VTT...), c'est à proximité du fond de vallée, des villages et de la station locale de sports d'hiver (Puy-Saint-Vincent) que ces espaces sont aménagés et intégrés à l'offre touristique.

Ajoutons enfin que cette dynamique est d'autant plus prégnante qu'elle est accentuée par deux mouvements convergents qui s'exercent aux deux extrémités de la vallée (figure 2) :

- A l'amont, la réglementation de la zone centrale du Parc National des Ecrins induit un certain nombre de restrictions qui tendent à rejeter en zone périphérique des activités comme le parapente, le VTT, voire l'escalade (convention portant sur l'équipement de voies nouvelles).

- A l'aval, l'Argentière-la-Besséc offre un véritable cas d'école de bourgcentre ayant fait l'objet d'un repositionnement touristique après une brutale désindustrialisation à la fin des années 1980. Cette reconversion volontariste est marqué par une recherche d'instrumentation des sports de nature comme nouveaux vecteurs économiques et identitaires el donc par l'appui sans réserve apporté à un ensemble de projets de développement : nouveaux sites de pratiques, installation de professionnels, organisation d'événements sportifs (open d'escalade sur blocs, internationaux de la cascade de glace).

Au-dela du cas exemplaire de la Vallouise, on retient que le développement des pratiques sportives de nature 
en périphérie des massifs (par double effet d'accessibilité et de rejet des espaces protégés), leur recentrage par rapport aux stations de sports $d$ 'hiver et aux villages de vallée (recherche d'animation et de diversification de l'offre), tout comme le repositionnement stratégique de bourgs-centres longtemps restés en dehors du jeu touristique, apparaissent comme de nouvelles constantes dans les figures de la « touristification $\approx$ qui se font jour depuis le début des années 1990.

\section{La dynamique du champ des métiers de la montagne}

L'approche des cultures professionnelles ne peut faire une impasse sui" la connaissance des évolutions et des changements qui alimentent le mouvement du marché des métiers du plein air. Les stratégies professionnelles s adaptent à cette dynamique en proposant de nouveaux produits. en s'inspirant des principes du marketing et en se pliant au jeu de la concurrence et aux nouvelles législations. Pour mieux comprendre les forces en présence dans le mouvement qui alimente ces changements, nous présentons ci-dessous quelques pistes qui semblent fécondes.

\section{Le choc des cultures professionnelles}

La multiplication des métiers du plein air et la segmentation des pratiques ont poul effet de modifier les profils professionnels. La spécialisation est dans 1'air du temps (techniciens, commerciaux, hôtesses. animateurs, gestionnaires...), tout comme la nécessitế pour l'indépendant de maîtriser des compétences multiples afin d'exercer sa profession. Les nouvelles formes de gestion et de commercialisation des structures de loisir imposent davantage de professionnalisme, tout comme on peut percevoir une tendance à voir augmenter la juxtaposition entre les grandes structures de prestation à orientation entrepreneuriale et celles des petites entreprises du plein air, très bien analysées par Malek Bouhaouala (2000). Le marché a tendance à se complexifier et cela d'autant plus quand augmente le poids des normes, des contraintes juridiques et de l'obligation de s"orienter vers une démarche $\nless$ qualité *

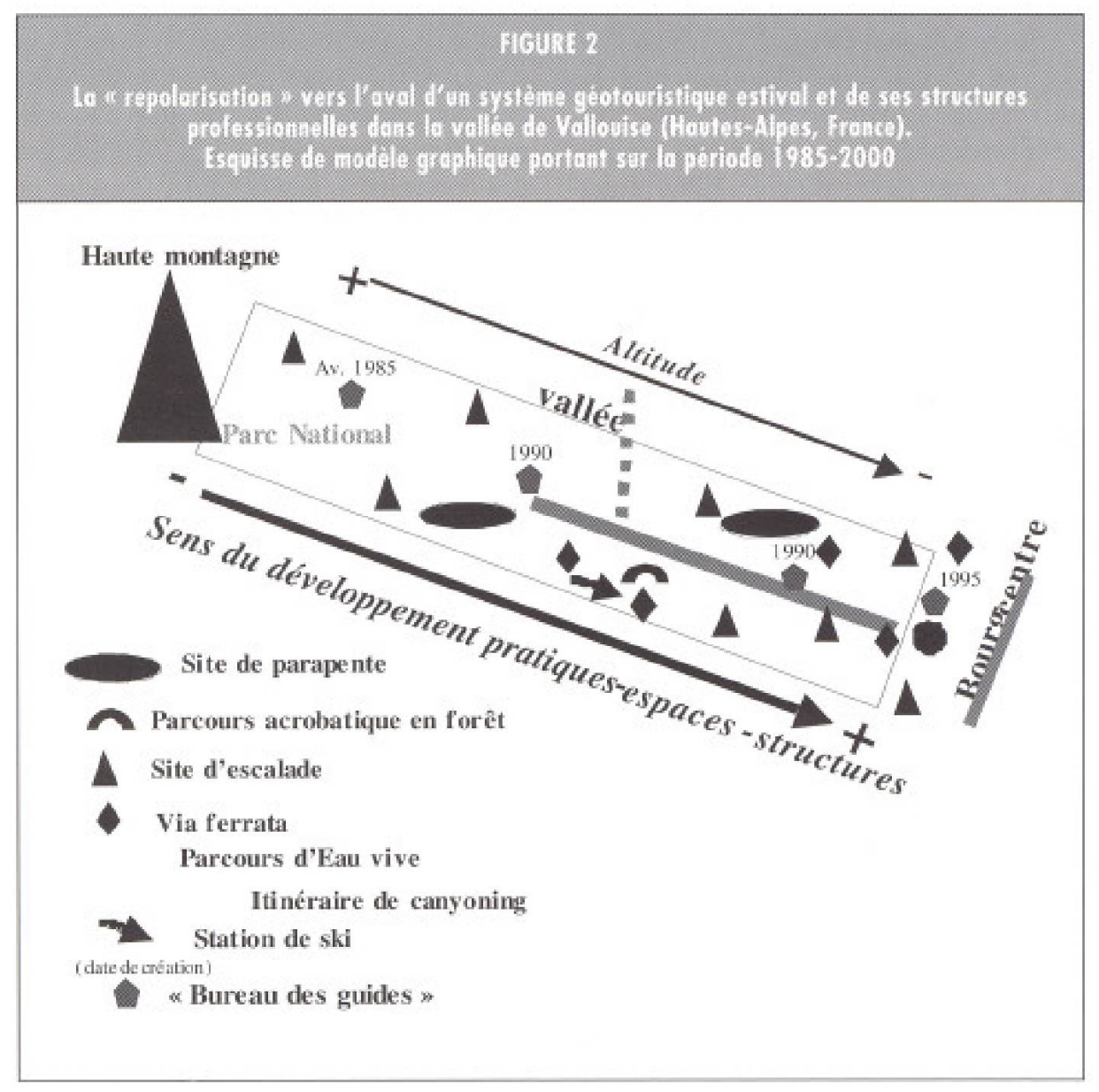

(Lambin, 1998). A l'behelle de l'ensemble du champ des métiers sportifs de la montagne conme a l'échelle d'une corporation, le maintien d'une culture professionnelle unifiée est de moins en moins évidente en présence de deux mouvements centrifuges : une fragmentation de l'intérieur et une ouverture vers les opérateurs de la grande 6 conomie dont les pratiques et les intérêts sont bien différents. La concurrence entre les cultures professionnelles s'en trouve exacerbée et de nombreux conflits inter ou intra-syndicaux illustrent les tensions qui naissent, non seulement sur le plan économique, mais aussi, de plus en plus, sur un terrain culturel et identitaire clairement explicité comme tel. C'est par exemple le cas du profond conflit qui a éclaté au sein du Syndicat National des Guides dans les années 1996-1997 entre les promoteurs d'une pluriactivité sportive (canyoning. « via ferrata $* . .$. ) et les partisans d'un recentrage identitaire sur la haute montagne et l'alpinisme.

\section{Une tendance au changement dans les cultures sportives du plein air}

Les métiers du plein air sont confrontés a la dynamique des sports de nature qui inventent de nouvelles modalités de pratique, créent des rêgles du jeu inédites et investissent différemment les territoires de pratique. Un ensemble de facteurs, qui vont de l'augmentation de la pratique * horsstructures $*$, de la fragmentation des styles de pratique, de la création de matériels innovants, de l'arrivee de pratiquants aux cultures disparates, de la montée en puissance d'une pluriactivité à la production de symboliques inspirées de la culture urbaine post-modeme, participent à l'éclattement de la culture sportive de nature. La déstabilisation de l'unité de cette culture est d'autant plus flagrante quand émergent de a nouvelles $*$ pratiques au caractère technique et culturel hybride : le vélo tout terrain (entre les univers du cyelisme et de la randonnée), la raquette à neige (entre 
les univers de la a glisse w et de la randonnée) et surtout le canyoning (entre les univers de la spéléologie, de l'eau vive, de la randonnée et de l'escalade). Cellesci font figure de cas d'école. Elles remettent en question les équilibres fragiles entre les prérogatives des fédérations sportives et celles des groupes professionnels concernés.

Alors que des décalages sont perceptibles entre l'univers des professionnels et celui des pratiquants, on peut observer des télescopages entre les cultures sportives ef le marketing du plein air, tout comme on peut observer la présence de concurrence entre logiques professionnelles. associatives, fédérales et individuelles. La où une unité était naguère perceptible, des tensions sont aujourd hui montantes, d'une part, entre usagers des espaces de nature et, d'autre part, entre les usagers et les professionnels du plein air ${ }^{17}$. On peut alors se poser la question de la place des métiers sportifs de la montagne dans cet espace de pratique et des relations qui se construisent actuellement entre ces deux univers.

\section{Une culture de l'innovation dans les produits sportifs de nature}

Le grand changement observable dans le champ des métiers du plein air semble se situer dans la montée en puissance de la culture de l'innovation par le marketing. Jamais autant que durant les quinze dernières annes, des innovations ont vu le jour et se traduisent par le développement et l'inflation de multiples produits. La creation de territoires sportifs marketing (parcs, sentiers, espaces ludiques, « via ferrata $^{18}, \ldots$ ), de prestations (événementiels, activites à theme, jeux de rôles, folklore, voyages en kit...), de matériels de tout genre (engins, sécurité, vêtements, objets technologiques...), sans oublier la transformation des formes de commercialisation s' inspirant largement des méthodes du marketing mix (service spécialisé, jeu vidéo, Intemet, promotion, stratégie clientèle et de fidélisation...), sont des exemples évocateurs. Toute cette dynamique fait entrer en force la culture du marketing et de l'innovation dans l'univers des métiers du plein air. Cela, alors que les réflexions produites par le Commissariat au Plan concernant le tourisme (1998) accordent une place centrale à l'information, à l'accès aux sites et aux pratiques, à la limitation des contraintes, aux produits insolites et artificiels (parcs...) pour répondre aux attentes de demain. Au-delà du professionnalisme attendu, c'est la prime à l'innovation et à l'imagination culturelle qui fera la difference (Corneloup, 2000 ; Le Goff, 1996). Sans aucun doute, on peut penser que le marketing devient aujourd' hui créateur de cultures professionnelles de nature innovante et participe grandement à la transformation des cultures sportives. La marchandisation du plein air est bien dans l'air du temps quand tous les espaces et toutes les pratiques sont l'objet d'une création ou d'une appropriation marketing Quelles limites est-il possible de reperer à cette culture de l'innovation dans le monde du plein air ? N'existe-t-il pas des résistances à la diffusion de cette culture marketing de la montagne, perceptibles au niveau des attentes de certains segments de clientèle? Une mise en perspective globale des modèles de management des espaces de nature (Corneloup, 1999b, 2000) montre l'existence de multiples solutions de remplacement aux développements marketing et entrepreneurial des territoires touristiques.

\section{Une tendance à I'éclatement de la clientèle de montagne}

Le changement des cultures professionnelles est évidemment lié à l'émergence d'une clientèle diversifiée, dont les segments semblent plus flexibles que par le passé. La multiplicité de ces segments, l'augmentation des exigences des consommateurs, la fidélisation qui s"effrite et la demande de pratiques plus consommatoires et ludiques participent à la recomposition des stratégies marketing. Sans doute, les clientêles de demain seront-elles de plus en plus éclatées (comités d'entreprise, jeunes de cités, troisième âge, étrangers, tourisme social, familles, handicapés...). Elles imposeront une adaptation constante des prestations et des produits. Il faudra aussi tenir compte du vieillissement de la clientèle, de la modification des demandes d'apprentissage (Pociello, 1985) et de la montée d'une culture sportive diversifiee qui auront pour effet de transformer la définition des produits, les attentes et les formes de satisfaction attendues (Loret, 2001). La culture du service ne fera que progresser au sein du monde du plein air, allant par exemple jusqu" considérer la sécurité et l'absence de danger comme un service. De multiples questions se poseront sur la manière de s'adapter à cette nouvelle clientèle et de lá séduire eu égard aux destinations concurrentes. Alors que des expertises tentent de saisir les tendances d'évolution des clientèles $\left(\mathrm{AFIT}^{19}\right.$, COFREMCA ${ }^{20}$. Foreseen, 1999b...), les fonctions culturelles du plein air et de la montagne devront s'adapter, changer et prendre en compte les demandes de naturalité, de vitalité, de ressourcement, de ludisme... qui semblent croissantes depuis le début des années 1990.

\section{La diversification des formes territoriales de gestion des espaces de montagne}

Les années à venir seront marquées par des enjeux importants autour des logiques de développement préconisées pour gérer les espaces sportifs de nature. Face à la montée de la question du tourisme durable et sous l'effet d'une tendance à voir augmenter la culture marketing et entrepreneuriale, le plein air aura à faire des choix pour proposer un développement adapté à la demande de la clientèle, à la politique étatique et aux exigences des professionnels et des intervenants locaux. Des luttes surviendront autour des questions de la fermeture / ouverture des espaces ; de la priorité accordée aux acteurs locaux sur les acteurs extérieurs et à la culture locale sur le monde des professionnels. Les écrits de Bernard Debarbieux ( 1995 ) et de Jean Paul Zuanon (2001) sur les formes de développement dans la vallée de Chamonix evoquent parfaitement cette problématique. De même, les incertitudes autour du tourisme durable ou éphếmêre alimenteront largement les débats sur le modèle de gestion légitime et acceptable (Corneloup. 2000). Les questions de la participation publique et de la gouvernance seront en débat, induisant des réflexions au sujet des formes de développement acceptables. 
Différentes formes ont été identifiées (fígure 3) ; elles participent à l'émergence du champ ou du marché des formes de développement au sein duquel des acteurs et des publics luttent pour imposer leur vision du monde, de la nature, de la politique et de l'économie (Comeloup, 2000). Trois méso-formes ont été identifiées autour desquelles s'engagent les acteurs professionnels : la méso-forme institutionnelle (formes republicaine, educative et technocratique), la méso-forme commerciale (marketing et entrepreneuriat) et la méso-forme locale (traditionalisme, développement local et écologie des profondeurs). Dans le cas de la méso-forme institutionnelle, l'action professionnelle est. pensée dans une optique publique à l'image des acteurs des parcs nationaux, du monde scolaire, des fédérations sportives et des services de l'État. Les activités sont des biens publics qui remplissent une fonction Educative, sociale et institutionnelle. Dans. la méso-forme locale, l'action s'inscrit dans l'univers local, On observe une perspective patrimoniale quand le poids des anciens et la volonté de respecter les triaditions s"imposent. Alors que l'action professionnelle vise à participer au developpement local (esthétisme, emploi de proximité, gestion de l'environnement, durabilite, adaptation aux changements), sur le plan de l'écologie des profondeurs, la passion, lá démarche alternative et la dissimulation dominent. Finalement, dans la méso-forme commerciale, les pratiques de la nature sont conçues comme des produits qu'il faut adapter à la clientèle ciblée et aux objectifs financiers de l'entreprise. La rentabilité, l'efficacité technologique, le service à la clientèle et la satisfaction du consommateur deviennent prioritaires.

Selon les cultures professionnelles des acteurs et les relations contractualisées, les formes de développement sont plus ou moins activées. Un style émerge, marquant l'identité du professionnel (Corneloup, 2000). L'enjeu porte aujourd'hui sur la capacité à produire des médiations et des traductions pour tendre vers un compromis acceptable entre les différentes parties présentes (Callon, 1986) dans des territoires touristiques de plus en plus

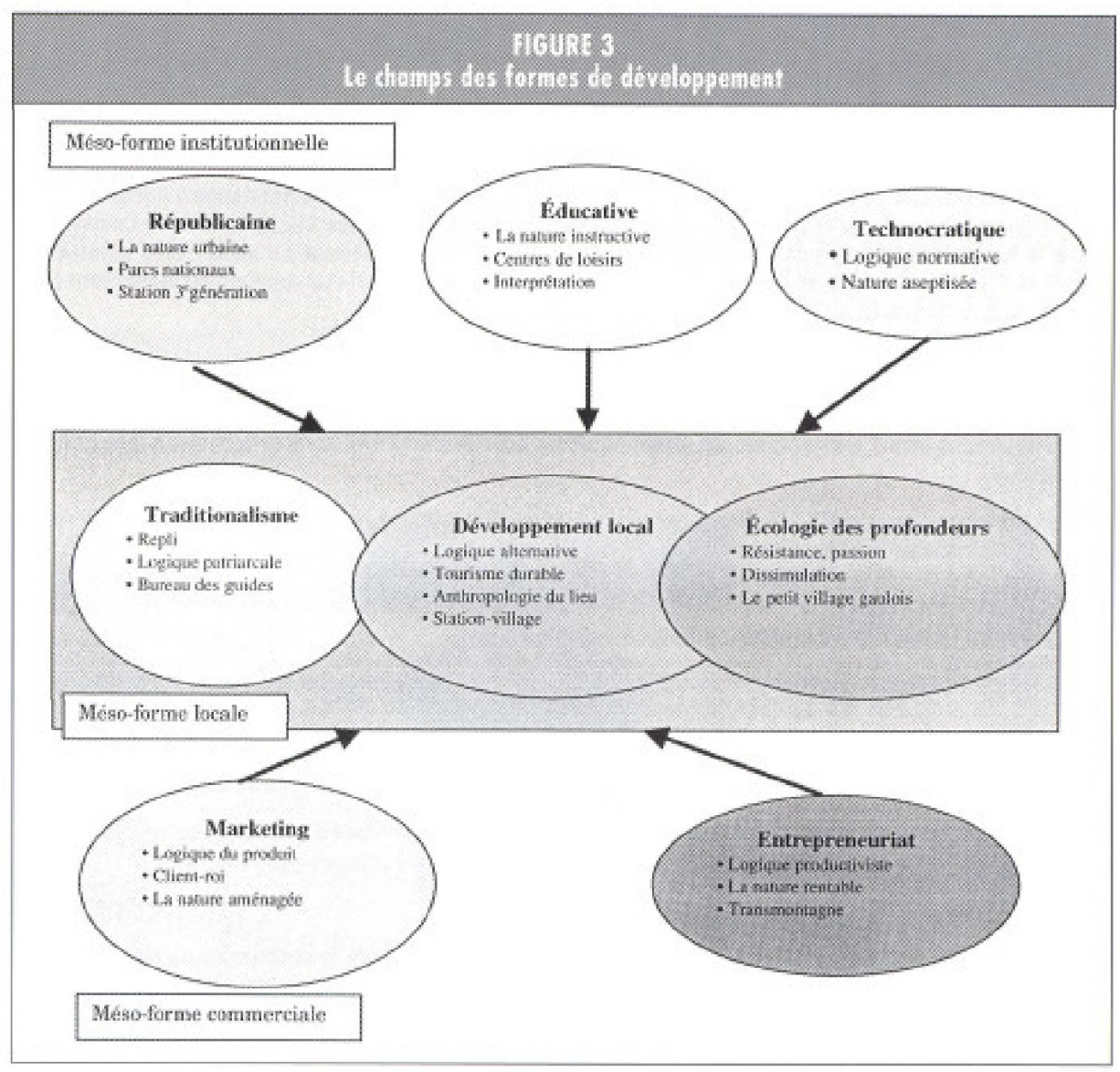

complexes. Pour Jean Viard (2000), l'approche par le territoire semble incontournable aujourd'hui, à la fois pour réaliser des diagnostics affinés et pour changer les formes de gestion. Le developpement de ce que cet auteur appelle une \& démocratie résidentielle $\%$ pour tendre vers des gestions de lieux de meilleure qualité semble nécessaire. Une fois de plus, la question de la participation est évoquée comme invitation à une meilleure coordination des actions développées par les acteurs attachés à des métiers touristiques au sein de territoires singuliers, par la mise en place d'une démocratie délibérative et participative.

\section{La modification des projets et des formations professionnelles}

Comment intégrer les changements dans la déclinaison des formations touristiques ? Bien souvent, l'approche des cultures professionnelles s'oriente vers l'analyse de la dynamique des métiers à partir d'une lecture verticale qui permet de saisir les grands changements et de donner quelques repères sur la structure montante. A l'image de la fiche technique sur les activités de pleine nature en Midi-Pyrénées, réalisée par le Centre de ressources pour les métiers du tourisme en espace rural (figure 4), ce type d'études semble bien insuffisant aujourd hui pour construire des formations adaptées à la dynamique du marché. L'établissement de programmes et de contenus de formation doit s'inscrire dans une mise en projet qui repose sur plusieurs variables dont il faut tenir compte, La consultation des formés (étudiants...) dans une optique participative, l'établissement d'une cartographie des métiers et des logiques d'action, l'étude du contexte professionnel dans lequel se situe la formation, la prise en compte des partenariats professionnels et institutionnels prévisibles et la définition de la stratégie de positionnement dans le champ des métiers du plein air s'imposent. Ces préalables permettent d'envisager la mise en place d'un programme de formation pragmatique en phase avec les situations d'action professionnelle vécues au quotidien dans le champ des métiers sportifs du plein 


\section{Ficunt 4}

Une logique do filitre demplai : fitite technicuse

sur les artivites de pleine noibre en Mith-Pyrentes

A partir d'une étude de 768 structures findividus ou emploveurs) proposant une prestation d'encadrementenseignement des activités de pleine nature en région Midi-Pyrénées, la CCI (Chambre do Commerce et d'Industrie) de Millau met en perspective un certain nombre de constats d'autant plus éclairants qu'il est rare de disposer de donnés approfondies à une vaste échelle territoriale qui associent des espaces montagnards et ruraux.

\section{La dynamique des structures}

- Alors que les deux tiers des structures concernées ont été créées depuis 1990, le rythme de création de nouvelles structures connait un falentissement qui paurrait caractériser l'arrivée à maturité du marché.

- Les statuts associatif ( $32,2 \%$ des structures), indépendant $\mid 30,3 \%)$ et de société commerciale $(14,2$ W) saont les trois profils dominants en matière d'encadrement sportif. Le statut indépendant connaît la plus forte croissance pendant les cinq dernières annèes, alors que le statut associatif semble en régression : le statut commercial est stable at semble quant â lui s'inserife plus dans une logique d'évolution structurelle que de création d'activités.

- $78 \%$ des structures disposent d"au moins un équipement complémentaire à l'encadrement: local d'accueil du public (64 \%), local technique (51 \%), hébergement (32\%), restauration (21 \%)

- $54 \%$ des structures fonctionnent á l'année, $19 \%$ sur une n double saison $n, 19 \%$ sur une saison $\%$ allongêe w de trois à cing mois, et $8 \%$ sur une saison a étroite $\%$ de un à deux mois.

- Le chiffre d'affaires annuel moyen par structure est de $316000 \mathrm{FF}$, avec une forte variabilite vers le haut qui bénéficie aux structures les plus anciennes et aux structures commerciales.

\section{Les activites et les prestations proposées}

- Les activités sportives proposées font l'objet d'une nette hiérarchie : randonnées pédestres $(45 \%$ des structures), randonnées équestres (44\%), eau vive $(33 \%)$, escalade-alpinisme-spéléologie $(24 \%)$. VTT $(22 \%)$, activitês aériẹnés (10\%).

- La logique d'offre est largement marquée par la pluriactivite : $65 \%$ des structures proposent au moins deux activités et $26 \%$ en proposent plus de cinq.

- La location de matériel sportif est proposée par 24 ớ des structures.

- 54 \% des structures proposent des prestations a l'heure, 77 \% a la demi-journée, $85 \%$ a la journée. $57 \%$ en sejours de moins de trois jours et $59 \%$ proposent d'autres types de séjours.

- $69 \%$ des structures proposent plusieurs activités dans un mẹme produit : une activité sportive et une activité découverte (48\%), plusieurs activités sportives ( $38 \%$ ).

- $45 \%$ des structures s'adressent ả une clientèle autant individuelle que collective, mais plus l'activité sportive proposée est technique lescalade, spéléo. VTT, voilel. plus la clientèle des groupes est importante (en escalade, par exemple, 34 \% des structures ont une clientẻle principalement composée de a groupes a contre $16 \%$ une clientèle principalement individuelle).

\section{L'organisation professionnelle et le partenariat}

- Dans le cas des activités sportives $\propto$ montagne et spáléo $n, 65 \%$ des structures adhèrent á un syndicat professionnel

- $75 \%$ des structures s associent avec des at hébergeurs $\%, 60 \%$ avec d'autres professionnels des sports de pleine nature, $38 \%$ avec des établissements de restauration, $32 \%$ avec divers prestataires locaux (animation, musées...).

- Le taux de monoactivité des professionnels concernés est de 33 \% pour les secteurs v escalade. alpinisme, spéléo $\mathrm{n}$ et $\mathrm{k}$ eau vive $\mathrm{n}$, de $22 \%$ pour le secteur a randonnée pédestre $\mathrm{x}$ et de $5 \%$ pour le secteur " randonnée équestre $\mathrm{n}_{\text {. }}$

\section{Les emplois}

- Le nombre d'emplois concerné est évalue à 3000 , soit 3.8 en moyenne par structure ( $59 \%$ d'hommes; äge moyen de 34 ans ; $60 \%$ d'emplois salariếs : $52 \%$ d'emplois â l'annẻe).

- La polyvalence iplusieurs composantes dans le poste de travail : encadrement, animation, organisation, gestion, commercialisation) concerne $53 \%$ des emplois.

- Trois profils de poste se dégagent : responsable d'activité (enc adrement et organisation), moniteur technicien (encadrement, entretien matériel et infrastructures) et responsable de structure (gestion, encadrement, commercialisation).

- $48 \%$ des employes d'encadrement-enseignement sont titulaires d'un BEES, $7 \%$ d'un BAPAAT, $24 \%$ d'un diplôme fédèral.

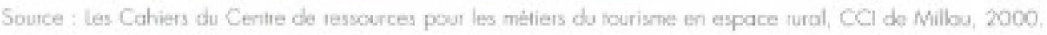

air. L'acquisition de compétences ne repose plus, dès lors, sur l'assimilation de savoirs et de savoir-faire " théorisés $*$, mais sur la maîtrise de savoirs operratoires qui se situent à l'interface entre les savoirs expérientiels et conceptuels (Dauberville, 1996). L'enjeu aujourd'hui se situe dans la capacité à évaluer les registres de compétences nécessaires pour permettre aux professionnels et aux étudiants de s'engager le plus possible dans le marché du tourisme sportif de montagne.

Dans cette perspective, un projet de formation se situe au carrefour de deux pôles structurants : les formes d'intervention pragmatique identifiées et les stratégies d'action et de développenent acceptables. Le premier pôle définit son programme d'action à partir de l'identification de référentiel de compétences et des logiques d'action expertisées. En s'appuyant sur les recherches effectuées en anthropologie cognitive (Ria, 2001 ; Gal et Durand, 2002) qu'il s'agit d'adapter à la connaissance des cultures professionnelles touristiques, les formes d'intervention, utilisées par les professionnels en situation, seront mieux identifiées. Par des approches de terrain prenant pour guide la grille d'expertise présentée précédemment (figure 1), une connaissance plus fine des logiques d'action et des compétences en ingénierie touristique est possible. La manière de concevoir la formation en sera bouleversée... Le deuxième pôle permet de situer la formation dans la dynamique des mé. tiers en intégrant les questions de l'éthique, du marketing stratégique et du politique. À partir du moment où l'on accepte l'idée que toute formation s"engage dans une ou des formes de développement \& implicatrices (figure 3), le détour par ce deuxième pôle s'impose pour favoriser la diffusion et l'acquisition de compétences réflexives et stratégiques.

Cette manière d'agir accorde toute sa place à un management et à une formation anthropologique (Corneloup, 1999b) permettant de ne pas se couper des cultures professionnelles, présentes et à venir, telles qu'elles prennent vie et se forment dans le quotidien de l'action " contextuée s. Une autre façon d'aborder les formes 
d'apprentissage dans l'univers des formations en tourisme sportif est évoquée, ce qui nécessite de fáire des études approfondies pour mieux saisir les cours d'action et d'apprentissage (Theureau, 2000).

\section{En conclusion}

Notre objectif consistait à proposer un modèle global pour analyser la dynamique des cultures professionnelles dans les sports de nature. Quatre pôles ont été identifiés autour desquels se structure historiquement cette dynamique. Celle-ci est aujourd' hui marquée par une mise à distance des relations historiques qui organisaient les échanges entre le pồle des cultures sportives de montagne et celui de l'action professionnelle en situation. De même, la formation professionnelle tend à s'ouvrir vers de nouveaux registres de compétences dépassant de bien loin ceux qui sont issus de la culture sportive. La dynamique des pôles étant poussée par le marche, la culture marketing tend à s'imposer dans le développement des produits sportifs de nature. En ce qui concernant la création de produits innovants en montagne, la culture sportive n'est plus motrice, mais semble se développer en parallèle au mouvement de la culture professionnelle. Par exemple, si les * via ferrata * implantées en France à partir de 1988 dans une logique de diversification de l'offre touristique ont rapidement fait l'objet d'une appropriation culturelle par les * montagnards *, cela ne semble plus guère le cas des parcours acrobatiques en forêt qui se multiplient depuis 1999. Cette rupture participe à l'ouverture du système vers d'autres logiques de jen et de fonctionnement : le directeur d'une station de ski, le responsable marketing d'un centre de loisirs, le concepteur de produits (objets, parcs aventure, événementiels...) ne sont plus nécessairement issus du monde et de la culture sportive de la montagne. Les métiers de terrain (guide, accompagnateur, moniteur kayak,...), s'ils ont souvent joué un rôle pionnier dans l'apparition des innovations observées, ne représentent plus qu'un pôle et qu'un segment des métiers du tourisme et du loisir sportif en montagne. Le marché se déplace vers d'autres acteurs et d'autres logiques de dévelop- pement qui modifient les logiques du jeu et les jeux de pouvoir. Une culture de la compétence s'impose en tant que nouveau modèle de formation aux métiers des sports de montagne, s'inspirant des principes émergents au sein des entreprises de demain pour gérer les ressources humaines actives et novatrices (Jolis, 2000).

Jean Corneloup est Maitre de conference a I'UFR STAPS de ClermontFerrand (France), II vient de teminer la rédaction du livre Les théries sociologiques de la pratique sportive (sous presse). ll est co-fondateur du reseau des chercheurs et des experts en sports de montagne et de nature.

Philippe Bourdeau est Maître de conférence à l'Institut de Géographie Alpine à Grenoble. Il a ccrit plusieurs lives sur l'histoire de l'alpinisme et le métier des guides de montagne. Il est aussi co-fondateur du réseau des chercheurs et des experts en sports de montagne et de nature (http.//www, multimanai,com/ sportsnature,fr).

\section{Notes}

1 Ecolle Nationale de Ski et d'Alpinisme.

2 Centres d'Éducation Populaire et de Sport.

3 . Le principe pour une caravane avec guide est que la décision appartient en toute circonstance à celui-ci. Lui seul jugera si lat course est possible ou non, s'il se présente tel ou tel danger, si tel passage est faisable ou non, s'il faut continuer ou faire demitour devant le mauvais temps... II lui appartient done d'avoir une fermete de caractere suffisante pour imposer sa wolonte à ses clients... x (Manuel d'alpinisme du CAF, 1934).

4 On rejoint ici les ecrits d'Alain Loret (1995) lorsqu'il évoque les principes de la culture digitale, cette culture orientée vers la maitrise de la nature, sa domestication ef vers la priorité donné à une seule culture sportive, celle des grandes pratiques classiques (alpinisme, randonnée...). En alpinisme, la seule pratique légitime chę les guides pendant plus d'un siècle a été la réulisation de courses en haute montagne.

5 Union Nationale des Centres Sportifs de Plein Air.

6 Club Alpin Français.

7 Union des Centres de Montagne.

8 Unité de Formation et de Recherche en Sciences et Techniques des Activites Physiques et Sportives.

9 Institut Universitaire Professionnalise.

10 Diplome d'études superieures specialisess.

II Les cerits de Jean Corneloup (1993) expliquent l'histoire de ces deux cultures, moderne (1800-1975) et post-moderne (1975-2000), dans l'etude des pratiques de l'cscalade. Durant la modernité, une seule culture est légitime et existe (l'alpinisme classique) ; avee la post-modernité, on entre dans l'ère du multiple avec un profond bouleversement des rapports à la nature, a la règle du jeu, aux espaces et aux symboles.

12 Le client est dans une logique securitaire passive quand c'est la technologie par le caible et l'aménagement * high tech * qui prennent en charge les erreurs possibles dans la mâtrise des risques par le pratiquant.

13 Dans son ouvrage sur les stations touristiques, Vincent Vlès (1996) montre, chiffres a l'appui, l'accroissement considérable du secteur \& Equipements et aménagements * $(+377 \%$ en dix ans $)$ dans les investissements touristiques.

14 L'usage d'Internet, des sites professionnels 


\section{Bibliographie}

Bernoud, Philippe (1996), * Sociologie des organisations ". Revue des sciences hwmaines. 64 , p. $46-51$,

Bouhaouala, Malek (2000), w Petites entreprises et territoires, le cas du secteur du tourisme sportif $*$, Tourisme sportif et territoires, Mon= tagnes méditerrancennes, 11, p. 41-47.

Bourdeau, Philippe (1998), " Les Alpes comme terrain de jeu de l'Europe *, Rapport sur l'État des Alpes, Commission Internationale pour la Protection des Alpes (CIPRA), Edisud. Aix-enProvence, p. 252-259.

Bourdeau, Philippe (1992), * Le touriste et son guide, Homo turisticus : du tourisme ordinaire en montagne $\%$, Revue de Géographie Alpine, 4/1991, Grenoble, p. 89-104.

Bourdeau, Philippe (1991a), L'escalade en France. Pratiques sportives et débouchés professionnels, Paris: La Documentation Française.

Bourdeau, Philippe (1991 b), Terrifoire et identité: recherches sur la territorialité d'un groupe professionnel, les guides de haute montagne en France, Grenoble : Collection Ascendance.

Bourdieu, Pierre (1987), Choses dites, Paris: Édition de Minuit.

Callon, Michel (1986), * Êléments pour une sociologie de la traduction $\%$, Année sociologique, vol. XXXVI.

Celard, Serge (1999), Rapport sur les conditions d'exercice de la profession de guide de haute montagne en 1998, Association pour la Recherche, l'Innovation et l'Adaptation en Montagne-Syndicat National des Guides de Montagne, Chambéry.

Chambre de commerce et d'industrie de Millau (2000), «es activités de pleine nature en MidiPyrénées ; analyse et conditions de développement d'une filière d'emplois *, Les Cahiers du Centre de ressources pour les métiers du tourisme en espace rural, Millau.

Corneloup, Jean (2000), « Les modèles de développement en management des espaces de nature *, Tourisme sportif et territoires, Montagnes médilerranéennes, 11, p. 95-99.

Corneloup, Jean (1999a), * Le communicationnel, un référentiel d'analyse des pratiques d'escalade durant la modernité , Histoire du sport. histoire des sportifs, L'Harmattan, p. $351-373$.
Corneloup, Jean (1999b), « Pour une autre approche managériale du sport $\%$, Gestion 2000 , Belgique, p. 33-60.

Dauberville, Les sciences humaines dans l'entreprise, Paris : Economica, 1996.

De Bellefon, R. (2001), * Paroles de guides... Identités de montagnards et d'alpinistes $*$, Babel, Hommes et monlagnes, 5, p. 81-102.

De Bellefon, R. (1997), Du terroir au territouire : histoire des guides de montagne en France (1870-1960). Thèse, Université de Toulouse.

Debarbieux, Bernard (1995), Tourisme et montagne, Paris : Economica.

Foreseen (Observatoire) (1999a), La soif d'émotion, Paris : Plon.

Foreseen (Observatoire) (1999b), Les nouveau horizons de la consonmation, Paris : Plon.

Gal-Petitfaux, Nathalie, et Marc Durand (sous presse), L'enseignement de l'Educasion physique comme " action situde : propositions pour une approche d'anthropologie cognitive. STAPS.

Hoibian, Olivier (2000), Les alpinistes en France, Paris : L'Harmattan.

Jeu, Bernard (1984), Le spon. l'émotion. l'es. pace, Paris : Édition Vigot.

Jolis, Nadine (2000), La competence au caur du succès de vorre entreprise, Paris : Edition d'organisation.

Lambin, Jean Jacques (1998), Le marketing stratégique, Paris : Édiscience.

Le Goff, Jean Paul (1996), Les illusions du management. Paris : La Découverte.

Loret, Alain (2000), \& Sport début de siècle : les mutations en cours et leurs conséquences stratégiques $\%$, Revue en management du sport. 3, p. $5-33$

Loret, Alain (1995), Géneration glisse, Paris : Autrement.

Loret, Alain (sous la direction de) (1993), Sport et management, Paris : Dunod.

Loux, Françoise (1988), Guides de montagne, mémoire et passions, Grenoble : Édition Didicr-Richard.

Maffesoli, Michel (1993), La contemplation du monde. Paris: Grasset.

Majastre, Jean Olivier et Decamp Éric (1988), Guides de haute montagne, Grenoble : Édition Glénat.
Midol, Nancy (1992), "Paradoxes de la dissidence s, Le corps surnaturé. Autrement. 4. p. 54-63.

Pigeassou, Charles (1997), Management des organisations de services sportifs, Paris : Presses universitaires de France.

Pociello, Christian (1995), Les cultures sportives, Paris : Presses universitaires de France.

Quéré, Louis (2000), « Singularité et intelligibilité de l'action *, L'analyse de la singularité de l"action, Paris: Presses universitaires de France, p. 147-170.

Ria, Luc, Jacques Saury, Catherine Sève, et Mare Durand (2001), * Les dilemmes des enseignants débutants : Études lors des premières expériences de classe en Education Physique \%, Science et Motricité, 42. p. 47-58.

Theureau, Jacques (2000), \& Anthropologie cognitive et analyse des compétences $m$ L'analyse de la singularité de l'action. Pat. ris: Presses universitaires de France, p. 171 . 212.

Vachée. Cécile (2000), Représentations de la nature et comporlements d'offre en escalade. Étude à partir de 3 catégories d'acteurs présidents de clubs, moniteurs brevetés d'Êtat et equipeurs de falaises. Thèse de doctorat STAPS, Université J. Fourier-Grenoble 1.

Viard, Jean (2001), Court traité sur les vacances, les voyages et l'hospifalité des lieux. La Tour d'Aigues, L'Aube intervention.

Viard, Jean (1998), Réinventer les vacances, Paris : La Documentation française.

Vlès, Vincent (1996), Les stations touristiques, Paris: Economica.

Zuanon, Jean Paul (2001), \& Le CAF et la vallé de Chamonix, 125 ans d'histoire w. Babel, Honmes et montagnes, 5. p. 161-174 